\title{
Focused Ion Beam Etching of Nanometer-Size GaN/AIGaN Device Structures and their Optical Characterization by Micro-Photoluminescence/Raman Mapping
}

\author{
M. Kuball ${ }^{1}$, M. Benyoucef ${ }^{1}$, F.H. Morrissey ${ }^{2}$, and C.T. Foxon ${ }^{3}$ \\ ${ }^{1}$ H.H. Wills Physics Laboratory, University of Bristol, Bristol BS8 1TL, UK \\ ${ }^{2}$ Philips Electron Optics BV, Eindhoven, Netherlands \\ ${ }^{3}$ Department of Physics, University of Nottingham, Nottingham NG7 2RD, UK
}

\begin{abstract}
We report on the nano-fabrication of GaN/AlGaN device structures using focused ion beam (FIB) etching, illustrated on a GaN/AlGaN heterostructure field effect transistor (HFET). Pillars as small as $20 \mathrm{~nm}$ to $300 \mathrm{~nm}$ in diameter were fabricated from the GaN/AlGaN HFET. Micro-photoluminescence and UV micro-Raman maps were recorded from the FIB-etched pattern to assess its material quality. Photoluminescence was detected from 300nm-size GaN/AlGaN HFET pillars, i.e., from the AlGaN as well as the $\mathrm{GaN}$ layers in the device structure, despite the induced etch damage. Properties of the $\mathrm{GaN}$ and the AlGaN layers in the FIB-etched areas were mapped using UV Micro-Raman spectroscopy. Damage introduced by FIB-etching was assessed. The fabricated nanometer-size GaN/AlGaN structures were found to be of good quality. The results demonstrate the potential of FIB-etching for the nano-fabrication of III-V nitride devices.
\end{abstract}

\section{INTRODUCTION}

Reactive ion etching [1] and wet etching techniques [2] have mostly been employed for the fabrication of III-V nitride devices with their wide spectrum of applications ranging from short-wavelength light emitters, solar-blind detectors to hightemperature devices [3,4]; these, however, are not the preferred techniques for the nanofabrication of III-V nitrides. Focused ion beam (FIB) etching is one of the most promising techniques for the fine patterning of III-V nitrides, however, only basic etching parameters have been investigated so far [5,6,7]. Alternatively, small nitride structures can be fabricated by selective area growth [8]. The direct write facility of FIB-etching - a well-established technique for optical mask repair and for IC failure analysis and repair allows the nanometer-scale fabrication of III-V nitride devices without the requirement for depositing an etch mask. A focused gallium ion beam of 5-20nm size is used in FIB to ablate the III-V nitride material. In this paper, we report on the nano-fabrication of a $\mathrm{GaN} / \mathrm{AlGaN}$ heterostructure field effect transistor (HFET) into pillars as small as 20$300 \mathrm{~nm}$ in diameter by FIB etching. The use of micro-photoluminescence and ultraviolet (UV) micro-Raman mapping to assess 300nm-size FIB-etched HFET structures is demonstrated. UV Raman scattering probes the vibrational states of the $\mathrm{GaN}$ and the $\mathrm{AlGaN}$ in the sample and provides information on the aluminum composition, the strain and the free carrier concentration in the fabricated structures [9-11]. Etch damage can easily be assessed from the photoluminescence and the Raman scattering intensity in the FIB-etched areas. 


\section{EXPERIMENT}

The GaN/AlGaN heterostructure field effect transistor (HFET) employed in this study was grown by molecular beam epitaxy (MBE) on a sapphire (0001) substrate, and consisted of a $25 \mathrm{~nm}$-thick low-temperature grown GaN buffer layer, a $1 \mu \mathrm{m}$-thick nominally undoped $\mathrm{GaN}$ layer, followed by a $3 \mathrm{~nm}$-thick nominally undoped $\mathrm{Al}_{0.15} \mathrm{Ga}_{0.85} \mathrm{~N}$, a $22 \mathrm{~nm}$-thick mid- $10^{18} \mathrm{~cm}^{-3}$ Si-doped $\mathrm{Al}_{0.15} \mathrm{Ga}_{0.85} \mathrm{~N}$, and a $15 \mathrm{~nm}$-thick nominally undoped $\mathrm{Al}_{0.15} \mathrm{Ga}_{0.85} \mathrm{~N}$ layer. The piezo-electric field effect combined with the intentional doping in the $\mathrm{Al}_{0.15} \mathrm{Ga}_{0.85} \mathrm{~N}$ layer contributes to the formation of a twodimensional electron gas at the $\mathrm{GaN} / \mathrm{Al}_{0.15} \mathrm{Ga}_{0.85} \mathrm{~N}$ interface investigated in [11]. Submicron machining was carried out on the samples using an FEI focused ion beam 2000 system. The FIB 2000 system uses a scanning $30 \mathrm{kV}$ gallium ion beam with an ion beam current in the range of $1 \mathrm{pA}$ to $1150 \mathrm{pA}$ and a spot size of $8 \mathrm{~nm}$ to $500 \mathrm{~nm}$, respectively, for the milling of the GaN and the AlGaN. Magnifications of the ion optics up to 150000 were used for scanning the gallium beam over the specimen. GaN/AlGaN pillars were etched at various beam currents and various magnifications of the ion optics. The fabricated structures were imaged using secondary electron imaging while scanning a gallium ion beam of low current over the sample surface. To evaluate the quality of the FIB-etched pattern micro-photoluminescence and micro-Raman spectra were recorded in $\mathrm{Z}(\mathrm{X}$, .) $\underline{\mathrm{Z}}$ geometry, i.e., unpolarized detection, using an ultraviolet (UV) Renishaw microphotoluminescence/Raman-system with a HeCd-laser (325nm) as excitation source. A 40x quartz objective was used to focus and collect the laser light with $1 \mu \mathrm{m}$ spatial resolution. The sample was scanned underneath the laser beam in $1 \mu \mathrm{m}$ steps using an $\mathrm{XY}$-stage to record photoluminescence and Raman maps of the FIB-etched areas.

\section{RESULTS AND DISCUSSION}

Figure 1 shows secondary electron images of pillars fabricated by FIB from the GaN/AlGaN HFET using gallium ion beam currents of 70pA, 4pA and 1pA, and magnifications up to 150000 . The images were recorded at an angle of $45^{\circ}$ with respect to the sample surface. A bitmap file of four square-shaped pillars was used as input file for the FIB 2000 system and defined the etched pattern. Figure 1(a) shows GaN/AlGaN pillars fabricated using a gallium ion beam current of $70 \mathrm{pA}$ and a magnification of 25000. The GaN/AlGaN pillars are square-shaped. They have a diameter of about $300 \mathrm{~nm}$ and a height of $0.9 \mu \mathrm{m}$. A gallium ion beam dose of $1500 \mathrm{pC} / \mu \mathrm{m}^{2}$ was used to achieve the shown etch depth. Smaller pillars of $150 \mathrm{~nm}$-size were etched at the same gallium ion beam current of 70pA using a magnification of 50000 and are shown in Figure 1(b). The pillars have a conical shape, corners of the pillars are round-shapes, illustrating a reduced etch quality. We note that beam currents of $70 \mathrm{pA}$ did not allow us to fabricate pillars smaller than $100 \mathrm{~nm}$ in size.

Using gallium ion beam currents of $4 \mathrm{pA}$ and $1 \mathrm{pA}$, sub-micron machining was carried out at magnifications of 100000 and 150000 , respectively. The results are shown in Figure 1(c) and (d). Pillars of 50-80nm diameter were etched at 4pA using a magnification of 100000 [Figure 1(c)]. Using a lower gallium ion beam current of 1pA and a higher magnification of 150000 , we succeeded in fabricating pillars as small as 20 $30 \mathrm{~nm}$ in diameter as displayed in Figure 1(d). The side walls are nearly vertical, illustrating the high quality of the fabricated pillars. The use of low beam currents is 

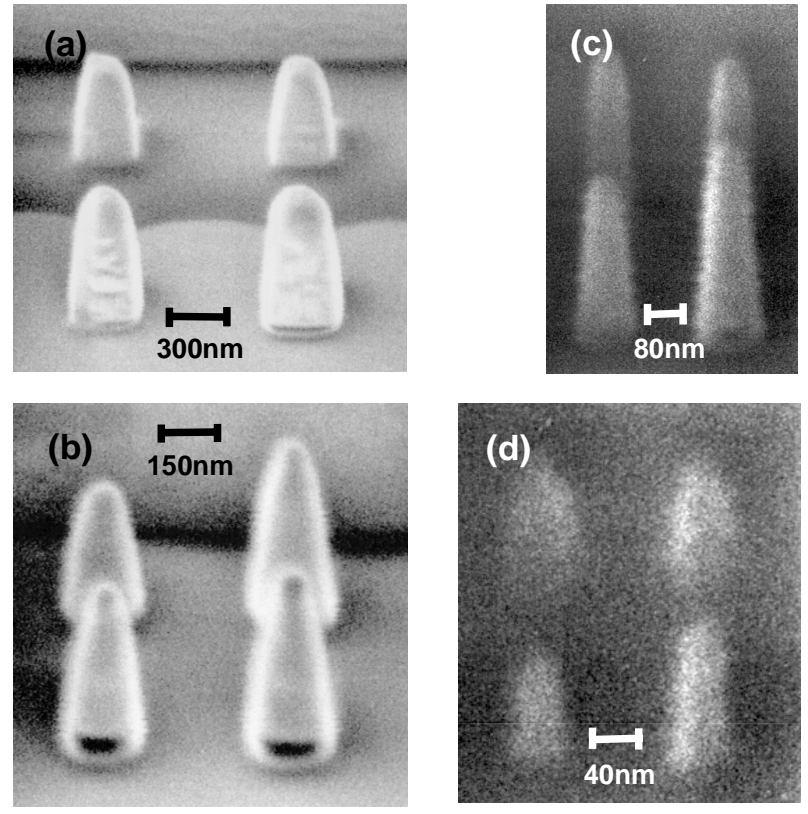

Figure 1. Secondary electron images of GaN/AlGaN-pillars FIB-etched (a) using a gallium current of 70pA at a magnification of 25000, (b) 70pA at a magnification of 50000, (c) 4pA at a magnification of 100000, (d) 1pA at a magnification of 150000.

essential to achieve a good fabrication quality using FIB [7]: the diameter of the gallium ion beam used for the milling decreases with decreasing current due to a reduced ion-ion interaction in the beam.

Micro-photoluminescence and ultraviolet (UV) micro-Raman spectra were recorded at room temperature from 300nm-size HFET structures similar to those shown in Figure 1(a) under $325 \mathrm{~nm}$-excitation. An area of about $10 \mu \mathrm{m} \times 10 \mu \mathrm{m}$ was removed around the pillars by FIB to allow access to their optical properties without interference from unetched areas of the sample. Figure 2(a) displays a spatial map of the $\mathrm{GaN} \mathrm{A}_{1}(\mathrm{LO})$ Raman intensity of the FIB-etched pattern. Each pixel of the map has a size of $1 \mu \mathrm{m} x$ $1 \mu \mathrm{m}$. The pillars are located in the center of the map and exhibit a large GaN Raman signal. The experiments probe all GaN/AlGaN pillars in the FIB-etched pattern at once. Figure 2(b) displays a map of the $\mathrm{Al}_{0.15} \mathrm{Ga}_{0.85} \mathrm{~N}$ photoluminescence intensity at $3.7 \mathrm{eV}$ (band gap of $\mathrm{Al}_{0.15} \mathrm{Ga}_{0.85} \mathrm{~N}$ [12]), i.e., $950 \mathrm{~cm}^{-1}$ shifted from the excitation laser line. The pillars emit $\mathrm{Al}_{0.15} \mathrm{Ga}_{0.85} \mathrm{~N}$ photoluminescence. Note the contour of the $10 \mu \mathrm{m} \times 10 \mu \mathrm{m}$ square etched around the pillars in Figure 2(a) and (b). Figure 3(a) displays a map of the $\mathrm{GaN}$ photoluminescence intensity at $3.38 \mathrm{eV}\left(3500 \mathrm{~cm}^{-1}\right)$. GaN photoluminescence is visible from the pillars. The large and asymmetric shape of the GaN photoluminescence area might be attributed to carrier diffusion. A spectrum recorded in the area of the pillars is shown in Figure 3(b). The FIB-etched 300nm-size GaN/AlGaN HFET pillars emit photoluminescence from the $\mathrm{Al}_{0.15} \mathrm{Ga}_{0.85} \mathrm{~N}$ and the $\mathrm{GaN}$ layers despite the induced etch damage. 

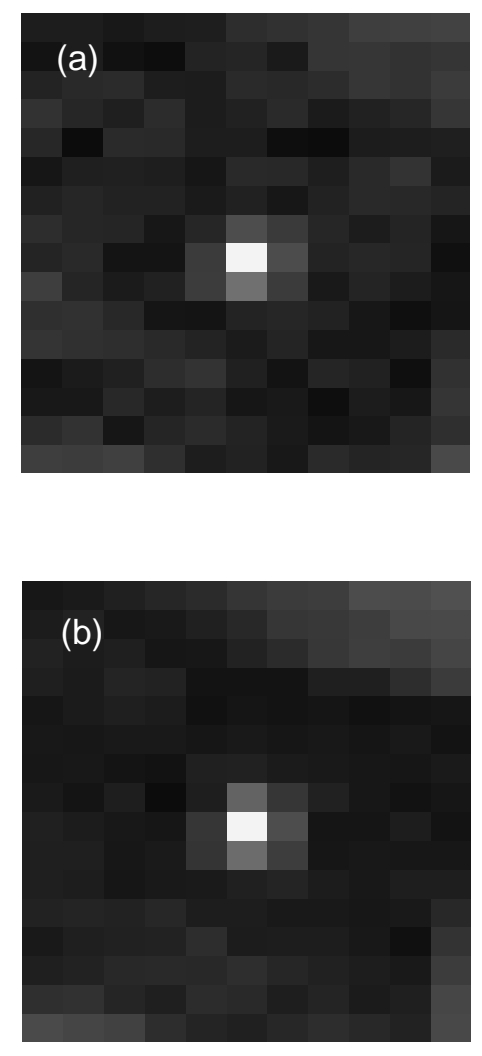

Figure 2. Micro-photoluminescence and UV micro-Raman maps of the FIB-etched GaN/AlGaN pattern of (a) the GaN $A_{I}(L O)$ Raman intensity at $734 \mathrm{~cm}^{-1}$ and (b) the $A l_{0.15} G a_{0.85} \mathrm{~N}$ photoluminescence signal at $3.7 \mathrm{eV}$ $\left(950 \mathrm{~cm}^{-1}\right)$. The pixel size is $1 \mu \mathrm{m} x$ $1 \mu \mathrm{m}$. The pillars are located in the center of the map. A Raman spectrum recorded on the pillars is shown in (c), one recorded adjacent to the FIB-etched area (secondary electron imaged to focus the ion optics prior to the FIB-etching) in (d), one of the sample before etching and imaging in (e).
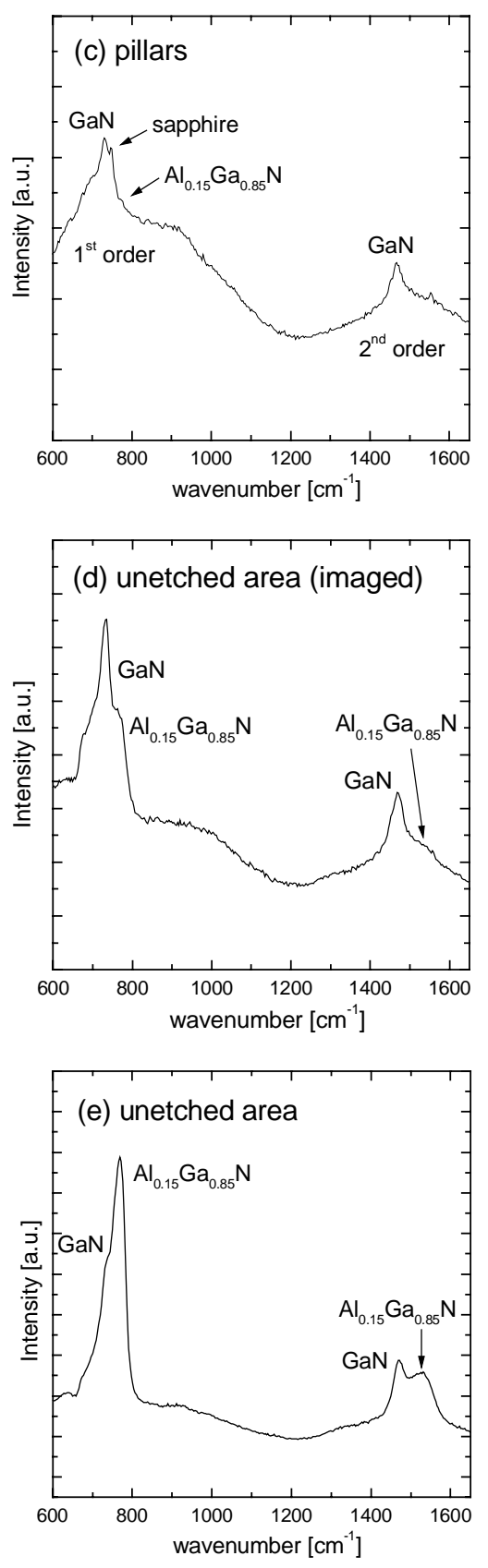

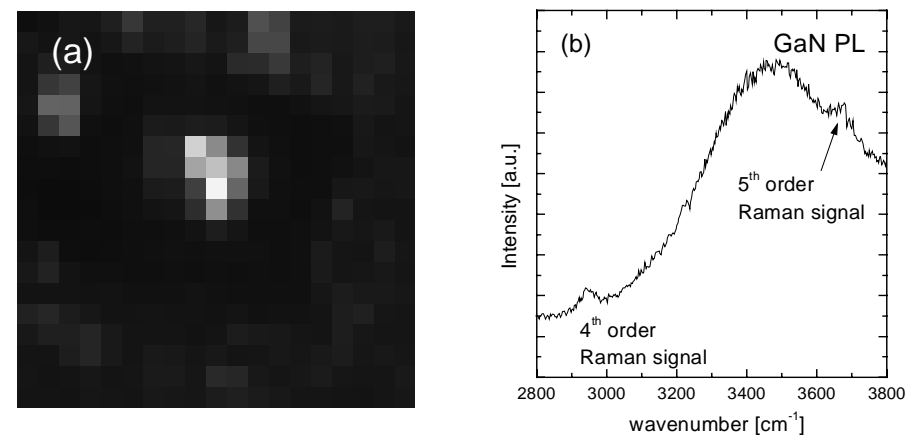

Figure 3. (a) GaN photoluminescence map at $3.38 \mathrm{eV}\left(3500 \mathrm{~cm}^{-1}\right)$ of the FIB-etched GaN/AlGaN pattern. The pixel size is $1 \mu \mathrm{m} \times 1 \mu \mathrm{m}$. The pillars are located in the center of the map. (b) Spectrum recorded in the area of the pillars.

Spectra recorded at different locations of the FIB-etched pattern are compared in Figure 2(c)-(e) to investigate in detail the damage introduced into the GaN/AlGaN HFET by FIB-etching. Figure 2(c) shows a Raman spectrum recorded on the pillars, Figure 2(d) one recorded adjacent to the FIB-etched area (secondary electron imaged to focus the ion optics prior to the FIB-etching). A Raman spectrum of the sample before imaging and etching is displayed in Figure 2(e). First-order Raman scattering from the GaN and $\mathrm{Al}_{0.15} \mathrm{Ga}_{0.85} \mathrm{~N} \mathrm{~A}_{1}(\mathrm{LO})$ phonons is visible in Figure 2(e) at $734 \mathrm{~cm}^{-1}$ and $771 \mathrm{~cm}^{-1}$, respectively, second-order Raman scattering at $1466 \mathrm{~cm}^{-1}$ and $1532 \mathrm{~cm}^{-1}$, respectively. The $325 \mathrm{~nm}$-excitation close to the bandgap of $\mathrm{GaN}$ and $\mathrm{Al}_{0.15} \mathrm{Ga}_{0.85} \mathrm{~N}$ results in the resonant enhancement of Raman scattering from the $\mathrm{A}_{1}(\mathrm{LO})$ phonons. The Raman modes are located on top of the $\mathrm{Al}_{0.15} \mathrm{Ga}_{0.85} \mathrm{~N}$ photoluminescence [11]. Due to the absorption of the laser in the $\mathrm{GaN}$ and $\mathrm{Al}_{0.15} \mathrm{Ga}_{0.85} \mathrm{~N}$ only a thin surface layer is probed in the experiments.

Imaging prior to the FIB-etching (Figure 2(d)) introduces damage in the surface layer of the sample. The $\mathrm{Al}_{0.15} \mathrm{Ga}_{0.85} \mathrm{~N}$ Raman intensity decreases. We also find a reduction in the $\mathrm{Al}_{0.15} \mathrm{Ga}_{0.85} \mathrm{~N}$ photoluminescence intensity. Damage introduced during the FIB-etching is illustrated by the decreased $\mathrm{Al}_{0.15} \mathrm{Ga}_{0.85} \mathrm{~N}$ Raman signal in Figure 2(c), present at $772 \mathrm{~cm}^{-1}$ as shoulder on the high-energy-side of the $\mathrm{GaN} \mathrm{A}_{1}(\mathrm{LO})$ Raman mode at $733 \mathrm{~cm}^{-1}$. We note that the shape of the pillars with their rounded top edges (Figure 1(a)) reduces the volume of the $\mathrm{Al}_{0.15} \mathrm{Ga}_{0.85} \mathrm{~N}$ with respect to the $\mathrm{GaN}$ and contributes to the reduction of the $\mathrm{Al}_{0.15} \mathrm{Ga}_{0.85} \mathrm{~N}$ Raman signal in Figure 2(c). To minimize this effect lower gallium ion beam currents could be used for the FIB-etching of the pillars. A close inspection of the $\mathrm{GaN}$ and $\mathrm{Al}_{0.15} \mathrm{Ga}_{0.85} \mathrm{~N} \mathrm{~A}_{1}(\mathrm{LO})$ phonon frequencies in Figure 2(c)-(e) reveals no significant frequency change $\left(<2 \mathrm{~cm}^{-1}\right)$ induced by imaging or etching, i.e., no large changes occur in the free carrier concentration, the strain and the aluminum composition of the $\mathrm{GaN}$ and $\mathrm{Al}_{0.15} \mathrm{Ga}_{0.85} \mathrm{~N}$ layers during the fabrication. Note the sapphire $E_{\mathrm{g}}$ Raman mode at $750 \mathrm{~cm}^{-1}$ in Figure 2(c). It is detectable after FIB-etching since the laser light $(325 \mathrm{~nm})$ can penetrate to the substrate between the pillars.

The 300nm-size HFET structures, i.e., the $\mathrm{GaN}$ and the $\mathrm{Al}_{0.15} \mathrm{Ga}_{0.85} \mathrm{~N}$ layers in the device structures, emit photoluminescence as shown in Figure 2(b) and 3(a) despite the etch damage illustrated in Figure 2(c)-(e), and show distinct Raman modes for GaN and $\mathrm{Al}_{0.15} \mathrm{Ga}_{0.85} \mathrm{~N}$ (Figure 2(a) and (c)) with frequencies, which were not affected by the nanofabrication. We can therefore conclude that the GaN and AlGaN layers in the FIB-etched 
device structure are of reasonable good quality, illustrating the potential of FIB for the nano-fabrication of III-V nitride devices. Processing steps to improve the FIB nanofabrication by reducing etch damage, however, need to be explored. This could involve the deposition of a protective film on the sample surface prior to the FIB-etching and the high-temperature annealing after the FIB etching. We note that FIB is a very timeconsuming process: FIB etching is a serial fabrication process in contrast to reactive ion etching (RIE). Large area etching is therefore not practical, however, this limitation can be overcome by using FIB only for the post-fabrication of RIE-etched structures, so that only small areas need to be modified by FIB to achieve small III-V nitride structures.

\section{CONCLUSIONS}

We have fabricated 20-300nm-size pillars from GaN/AlGaN HFET structures using FIB etching. Micro-PL/Raman mapping showed that 300nm-size GaN/AlGaN HFETs fabricated by FIB emit photoluminescence and a Raman signal despite the induced etch damage, illustrating the potential of FIB for the nano-fabrication of III-V nitride devices without the requirement for an etch mask. Processing steps to reduce etch damage need to be explored as next step to improve FIB-etched III-V nitride device structures. Then, properties of smaller FIB-etched structures need to be investigated.

\section{REFERENCES}

[1] I. Adesida, C. Youtsey, A.T. Ping, F. Khan, L.T. Romano, and G. Bulman, MRS Internet J. Nitride Semiconductor Res. 4S1, G1.4 (1999).

[2] C. Youtsey, I. Adesida, L.T. Romano, and G. Bulman, Appl. Phys. Lett. 72, 560 (1998).

[3] S. Nakamura, M. Senoh, S. Nagahama, N. Iwasa, T. Yamada, T. Matsushita, H. Kiyoku, Y. Sugimoto, T. Kozaki, H. Umemoto, M. Sano, and K. Chocho, Appl. Phys. Lett. 72, 211 (1998).

[4] S. Yoshida and J. Suzuki, Jpn. J. Appl. Phys. 37, L482 (1998).

[5] C. Flierl, I. H. White, M. Kuball, P.J. Heard, G.C. Allen, C. Marinelli, J.M. Rorison, R.V Penty, Y. Chen, S.Y. Wang, MRS Internet J. Nitride Semicond. Res. 4S1, G6.57 (1999).

[6] I. Chyr and A. J. Steckl, MRS Internet J. Nitride Semicond. Res. 4S1, G10.7 (1999).

[7] M. Kuball, F.H. Morrissey, M. Benyoucef, I. Harrison, D. Korakakis, and C.T. Foxon, Phys. Stat. Sol. (a) 176, 355 (1999).

[8] J. Wang, M. Nozaki, Y. Ishikawa, M.S. Hao, Y. Morishima, T. Wang, Y. Naoi, and S. Sakai, J. Cryst. Growth 197, 48 (1999).

[9] F. Demangeot, J. Groenen, J. Frandon, M.A. Renucci, O. Briot, S. RuffenachClur, R.-L. Aulombard, MRS Internet J. Nitride Semicond. Res. 2, 40 (1997).

[10] H. Harima, H. Sakashita, S. Nakashima, Mat. Sci. For. 264-268, 1363 (1998).

[11] M. Kuball, J.M. Hayes, A. Bell, I. Harrison, D. Korakakis, and C.T. Foxon, Phys. Stat. Sol. (a) 176, 759 (1999).

[12] D. Brunner, H. Angerer, E. Bustarret, F. Freudenberg, R. Hopler, R. Dimitrov, O. Ambacher, and M. Stutzmann, J. Appl. Phys. 82, 5090 (1997). 